\title{
Europäischer Datenschutz im 21. Jahrhundert
}

Der Integrationsprozess der europäischen Staaten schreitet fort. Auch Nicht-Mitgliedstaaten der EU haben sich bereits in die EU-Dynamik eingeklinkt. Der Historiker Wolfgang Schmale hält in seinem Werk "Geschichte und Zukunft der Europäischen Identität" (2008) zutreffend fest, dass auch auftretende Krisen an dieser Entwicklung wenig ändern werden. Vor diesem Hintergrund kreisen die Themen im DuD Heft 9 um den Datenschutz in Europa, der nicht nur im Mittelpunkt der grundrechtlichen Ordnung in Deutschland, sondern nach dem Reformvertrag auch in dem der EU stehen soll. Der durch den Antiterrorkampf geschwächte europäische Datenschutz soll unter der neuen EU-Präsidentschaft wieder gestärkt werden. Nicht noch mehr Eingriffsbefugnisse für die Sicherheitsbehörden, sondern stattdessen mehr Datenschutz beim Austausch von Informationen steht auf der politischen Agenda - so Martin Valfridsson, Sprecher der schwedischen Justizministerin auf Anfrage von heise online (heise online, 17.7.2009). Auch in der Mitteilung der Kommission vom Juni 2009 („Ein Raum der Freiheit, der Sicherheit und des Rechts im Dienste der Bürger") ist von "neuen allumfassenden Maßnahmen zum Schutze der Daten der Bürger" die Rede. Zugleich wird die Frage neu diskutiert, ,' unter welchen Umständen Behörden in Ausübung ihrer rechtmäßigen Funktionen der Anwendung dieser Vorschriften gegebenenfalls Grenzen setzen könnten."

In der Vergangenheit wurden diese Grenzen mitunter sehr einseitig zulasten des Datenschutzes gezogen. Insbesondere die Richtlinie 2006/24/EG zur Vorratsdatenspeicherung ist trauriges Beispiel für eine neue Dimension europäischer Daten-Sammelwut. Der EuGH hat die nahe liegende Frage nach einer Vereinbarkeit der Richtlinie mit den Grundrechten allerdings offen gelassen (siehe Gateway). Michael Ronnellenfitsch setzt sich im ersten Beitrag differenziert und umfassend mit der Geltung des Grundrechts auf informationelle Selbstbestimmung im Verhältnis zum EU-Recht auseinander. Rainer Schweizer untersucht sodann den Datenschutz im Spektrum der Rechtsprechung des EGMR und führt die Bedeutung der EMRK als europäisches Fundament des Persönlichkeits- und Datenschutzes vor Augen. Hans-Hermann Schild und Marie-Theres Tinnefeld versuchen auch unter Berücksichtigung dieser Rechtsprechung den notleidenden Arbeitnehmerdatenschutz darzulegen, der durch die Neuregelung im BDSG keine wirklich neuen Impulse erhalten hat. Wie unterschiedlich Datenschutz auch innerhalb eines einheitlichen europäischen Rechtsrahmens ausfallen kann, veranschaulicht Burkhard Schäfer am Beispiel der Überwachung in Großbritannien. Benedikt Buchner wendet sich in seinen Ausführungen dem gläsernen Sportler zu, dessen Recht auf informationelle Selbstbestimmung im Zuge der weltweiten Anti-Doping Bemühungen ohne das Dagegenhalten der europäischen Seite völlig in den Hintergrund getreten wäre. Alexander Dix zeigt die Risiken auf, die dem Patientengeheimnis durch die zunehmend vernetzte Verarbeitung von medizinischen Befunden drohen - angefangen bei der Datenspeicherung auf Computern mit Internet-Anschluss (Problem der Online-Durchsuchung) bis hin zu GoogleHealth. Marie-Theres Tinnefeld befasst sich mit dem technologisch geprägten Grundrechtsschutz, vor allem mit den Fragen der Online-Durchsuchung und dem neuen Grundrecht auf Gewährleistung der Vertraulichkeit und Integrität von IT-Systemen. Tim Fickert behandelt offene Datenschutzfragen und unbefriedigende Antworten zum Thema Geodaten bei Google Street View - zu Recht weist der Landesdatenschutzbeauftragte Edgar Wagner mit Blick auf Google Street View darauf hin, dass die zunehmende Veröffentlichung von Privatheit und privatem Umfeld dringend einer gesellschaftlichen Diskussion bedarf. ${ }^{1}$

\section{Marie-Theres Tinnefeld, Benedikt Buchner}

1 http://www.datenschutz.rlp.de/de/presseartikel.php?pm=pm2009062502 